\title{
Erosion Damage to Solids Caused by High-Speed Collision With Rain*
}

\author{
Olive G. Engel
}

\begin{abstract}
The stresses that are produced when a liquid drop collides with the planar surface of a solid are discussed. These are a result of the impact pressure and the consequent radial flow of the drop. It is concluded that a rain-erosion resistant material may be either soft and rubbery or hard and rigid. If it is soft and rubbery it mitigates the applied stresses but it must be able to withstand the mitigated stresses. If it is hard and rigid it does not mitigate the applied stresses and it must be able to withstand the unmitigated stresses.
\end{abstract}

\section{Introduction}

The problem of the erosion damage that is produced on objects that fly at high speed into rain has become increasingly more serious as flight velocities have been increased.

During the past 10 years, a program of testing has been carried out to find materials that will resist the stresses that are brought to bear in high-speed collision with rain. The work has been done mainly at the Cornell Aeronautical Laboratory. Specimens of materials have been fastened to the ends of a propeller and have been rotated at velocities up to approximately 600 miles per hour (mph) through artificial rain. Tests of a different kind have been carried out at higher velocities by the Convair Division of General Dynamics Corporation.

During the past 5 years, research has been in progress at the National Bureau of Standards to determine the mechanism of the erosion process.

A previous publication ${ }^{1}$ has presented three factors that are important in analyzing the damage that is produced as a result of high-speed waterdrop collision with poly(methyl methacrylate): (a) The waterdrop behaves like a hard sphere or pellet in that it produces a cup-shaped cavity in the plastic; (b) although materials do not usually fail under uniform compression, a local compression can produce tensile stresses that result in failure; and (c) in the case of projectiles that flow as a result of the collision, the solid plastic can be damaged by the stresses exerted by the flowing projectile against surface irregularities that are restrained by the underlying layers of material.

\section{Collisions of Waterdrops with Aluminum}

Further evidence of the behavior of water under impact conditions was obtained in a study of the damage marks left by deforming lead pellets, steel spheres, and waterdrops as a result of high speed collision with soft 1100 aluminum sheet. Figure 1, A shows an example of a damage mark made by a $7 / 32$-in.-diam steel sphere on 1/8-in. 1100-H14 aluminum sheet. This particular damage mark was obtained at a relative impingement velocity of 228

*The work described in this paper was sponsored by the Wright Air Development Center. The material presented here has been taken largely from reports submitted to them. Their assistance and interest are gratefully acknowledged.

1 Olive Engel, J. Research NBS 54, 51 (1955) RP2565.

feet per second (fps). The diameter of this damage mark, as measured directly on the aluminum plate with a steel rule and magnifying glass, is about $4 / 32$ in., which is considerably less than the diameter of the steel sphere. Figure 1, B shows an example of a damage mark that was made by a 0.22 -in.-diam deforming lead pellet on 1/8-in. 1100-H14 aluminum sheet. This particular damage mark was obtained at a relative impingement velocity of $609 \mathrm{fps}$.

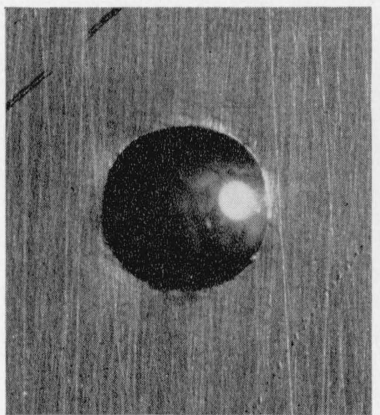

A
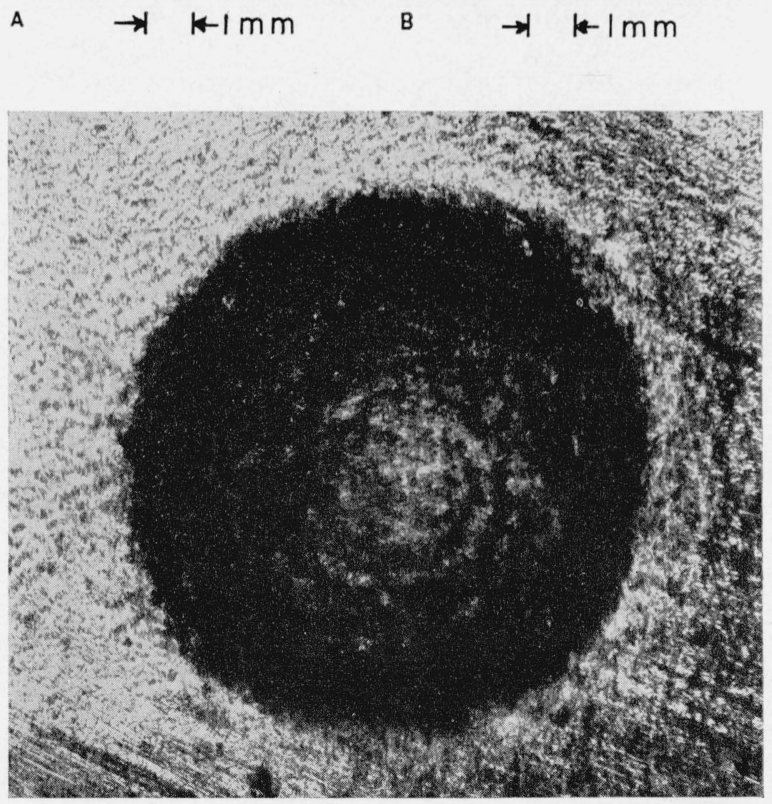

C

Figure 1. Damage pits in 1100 aluminum formed as a result of collisions with (a) a steel sphere at a velocity of $228 \mathrm{fps}$, (b) a lead pellet at a velocity of $609 \mathrm{fps}$, and (c) a waterdrop at a velocity of $2,500 \mathrm{fps}$.

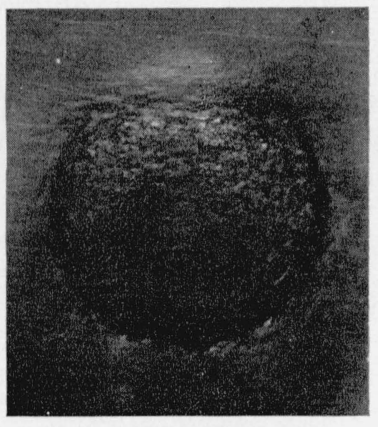

B $\rightarrow k-1 \mathrm{~mm}$

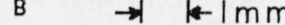


At a collision velocity of $880 \mathrm{fps}(600 \mathrm{mph})$, waterdrop damage marks that have been observed in 1100-O aluminum resemble the mark left in 1100-H14 aluminum by impingement of a deforming lead pellet at very low velocity. When a waterdrop collides with 1100 aluminum at about $2,000 \mathrm{fps}$, an impression is made which resembles that made by a low-velocity steel sphere more nearly than that made by a relatively low-velocity lead pellet (see fig. 1). These observations lead to the conclusion that in the range of collision velocity up to about 1,000 fps a waterdrop behaves like a projectile that flows as a result of the collision, but at collision velocities of the order of $2,000 \mathrm{fps}$ a waterdrop behaves very much like a projectile that does not flow as a result of the collision.

\section{Destructive Action of the Radial Flow of a Waterdrop}

When a waterdrop collides with the planar surface of a solid, or when the planar surface of a solid runs into a stationary waterdrop, the impact pressure that results reaches a high value in a very short time. ${ }^{2}$ This high pressure drives the liquid that is close to the solid surface radially outward around a central stagnation point. The radially flowing liquid exerts a shear stress on the surface of the solid over which it is running. There is a shear stress between the separate layers of the flowing liquid itself, and it can be expected that a shear stress exists at the interface between the liquid and a solid surface over which it is moving. The shear stress, $\tau$, between layers of liquid in laminar flow is given by the product of the viscosity, $\mu$, and the velocity gradient through the moving sheet of liquid perpendicular to its direction of flow. That is, $\tau=\mu(\partial v / \partial z)$ where $v$ is the velocity of the moving sheet of liquid and $z$ is the direction through the thickness of the liquid sheet. The layer of liquid molecules in direct contact with the solid has zero velocity, but the velocity gradient is not zero and the shear stress is applied to the solid.

When a surface protrusion exists, the liquid from a drop that is flowing radially over it exerts forces against the protrusion acting in opposition to the cohesion of the protrusion to the underlying layers of materia]. Pressure, $\sigma_{p}$ of figure 2 , is exerted against the protrusion by the flowing liquid. The pressure that is exerted by the liquid tends to move the protrusion along the planar surface of the solid and results in a shear stress, $\tau^{\prime}$ of figure 2 , at the base of the protrusion. The flow of the liquid results in the shear stress, $\tau$, that was discussed above. The pressure exerted by the liquid also results in a turning moment that tends to bend the protrusion over. The turning moment is the integrated cross product of the compressive force exerted by the liquid and the elevation of the protrusion above the planar surface of the solid at the point where the force is applied. As the protrusion bends, a tensile stress, $\sigma_{t}$ of figure 2, appears on the side of the protrusion

\footnotetext{
2 Olive Engel, J. Research NBS 54, 281 (1955) RP2591.
}

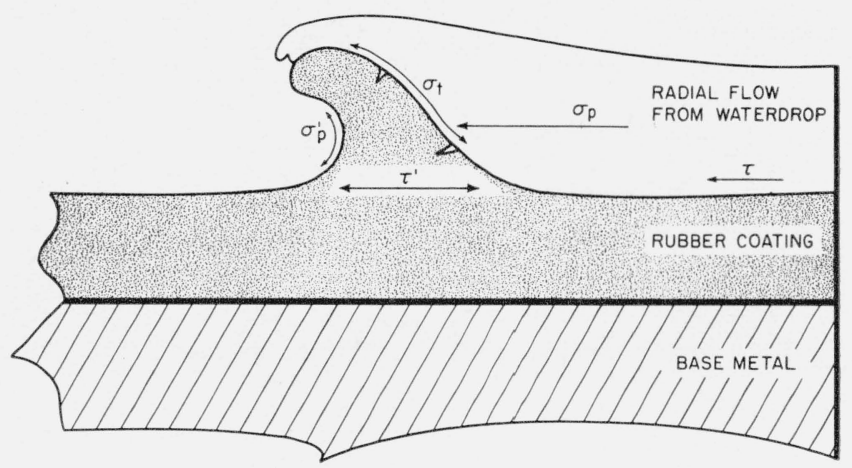

Figure 2. Schematic diagram of the stresses that exist in a surface protrusion of a rubber coating as the result of the radial flow of an impinging waterdrop.

against which the compressive force is applied and a compressive stress, $\sigma_{p}^{\prime}$ of figure 2, appears on the opposite side of the protrusion. If the force exerted by the rapidly running liquid is sufficiently great, or if the protrusion has a sufficient elevation above the planar surface, failure may occur. Whether the protrusion is simply bent over, whether it is broken off, or whether part of the solid material below the surface is torn out with it depends on the strength of the material and on where failure occurs first. Likely points of failure are marked with notches in figure 2.

If a waterdrop impinges against the planar surface of a rubberlike material the effect of the compressive stress, $\sigma_{p}$ of figure 3 , which is the direct result of the collision, is to compress the solid material at the point of impingement. The local compression results in a dimple or cup-shaped cavity. Tensile stresses, $\sigma_{z}$ of figure 3, appear in the sides of the dimple and especially in the knee in the solid material at the rim of the dimple. If these tensile stresses are sufficiently great, tears may form in the surface layers of the solid material. The material that formerly occupied the volume that now forms the hollow of the dimple is displaced. It is moved radially outward and upward around the dimple and this results in a shear stress, $\tau^{\prime}$ of figure 3 . The radial flow of the liquid of the drop imposes a radial tensile stress, $\sigma_{t}^{\prime}$ of figure 3 , and a shear stress, $\tau$, which was discussed above. In the case of very thin rubbery coatings it is possible that a portion of the shear stress, $\tau$, may be transmitted through the coating to the primer bonds,

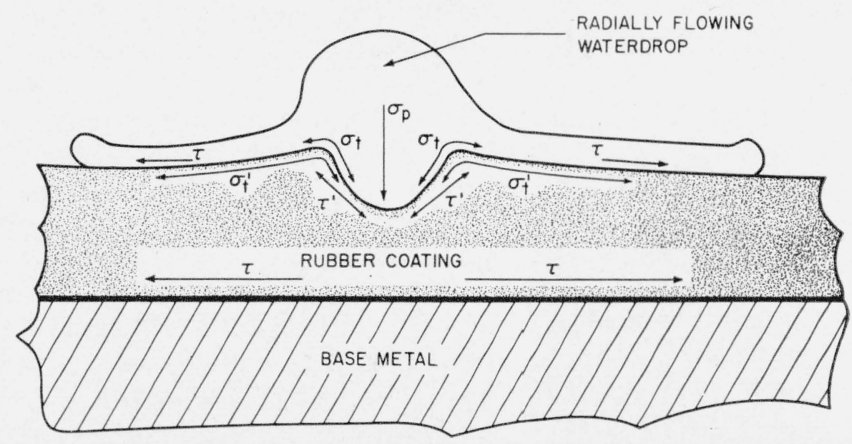

Figure 3. Schematic diagram of stresses that exist in a rubberlike coating as a result of the collision and flow of a waterdrop. 
which hold the coating to the base metal, and that it may cause failure of the primer-to-rubber or of the primer-to-metal bond. However, the removal of a circle of rubber coating around the central point of the collision (stagnation point of the flow), as occurred in the case of a Neoprene coating (see fig. 6), is not necessarily the result of transmission of this shear stress through the coating. It is possible that a deep circular cut may form as a result of the tensile stress, $\sigma_{t}$ of figure 3 , in the material at the rim of the dimple or cup-shaped cavity. If this cut extends through the coating the radially flowing liquid may lift the coating with a wedge action and peel it off.

\subsection{Waterdrop Traces in Fine Moist Sand}

The effect of the forces that come into play when the liquid of an impinging drop flows radially can be seen in the marks that are left when waterdrops fall into fine moist sand. To obtain the picture of such traces shown in figure 4, very fine mesh sand that was just moist was gently pressed into a large petri dish. The surface of the sand was leveled and was then lightly covered with carbon black to as uniform a degree as possible. Waterdrops were allowed to fall from a flat-nosed pipet through a distance of approximately $40 \mathrm{ft}$ to the surface of the carboncovered sand. The waterdrop traces in figure 4 show clearly the existence of the stagnation point at the center of the flow where the radial flow velocity is zero; at this point the carbon has not been washed from the surface of the sand. The circular trench about the stagnation point is the result of the forces that act as a consequence of the radial flow of the liquid. The relative collision velocity was about $25 \mathrm{fps}$.

\subsection{Waterdrop Traces Produced in a Neoprene Coating}

Traces similar to those that formed when waterdrops were allowed to fall into the petri dish of carbon-covered sand were also found on a Neoprene ${ }^{3}$ coated specimen that was rotated on the Cornell

3 This was not one of the standard Neoprene coatings that fulfilled the requirements of Specification MIL-C-7439.

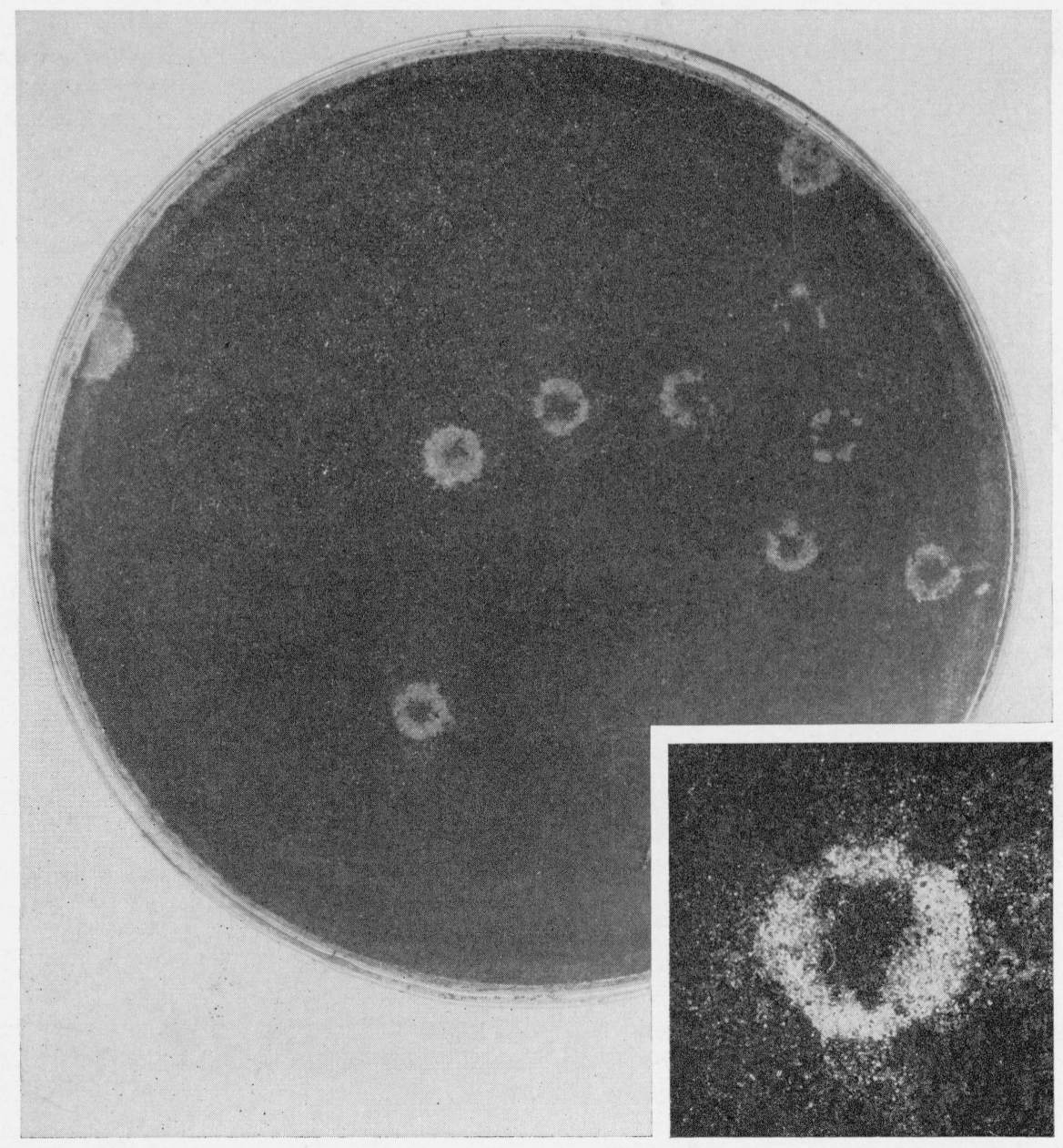

FIGURE 4. Traces of the radial flow of waterdrops that collided with sand that was dusted with carbon black.

The inset is a magnification of one trace. 
Aeronautical Laboratory rain erosion test apparatus at a velocity of $600 \mathrm{mph}$ through 1-inch per hour (iph) artificial rain for $1.5 \mathrm{~min}$ (see fig. 5). The average coating thickness was 8 to 10 mils. Because the surface is not completely covered with such damage marks it seems likely that traces of this kind are not produced by the first waterdrop collision with the unfatigued Neoprene coating. They are probably formed during a single collision but only after the coating has suffered deterioration of some kind (possibly permanent set) as a result of numerous radial-stretch-and-recover cycles imposed by the radial flow of waterdrops that collided earlier but that were not themselves able to produce the observable damage.
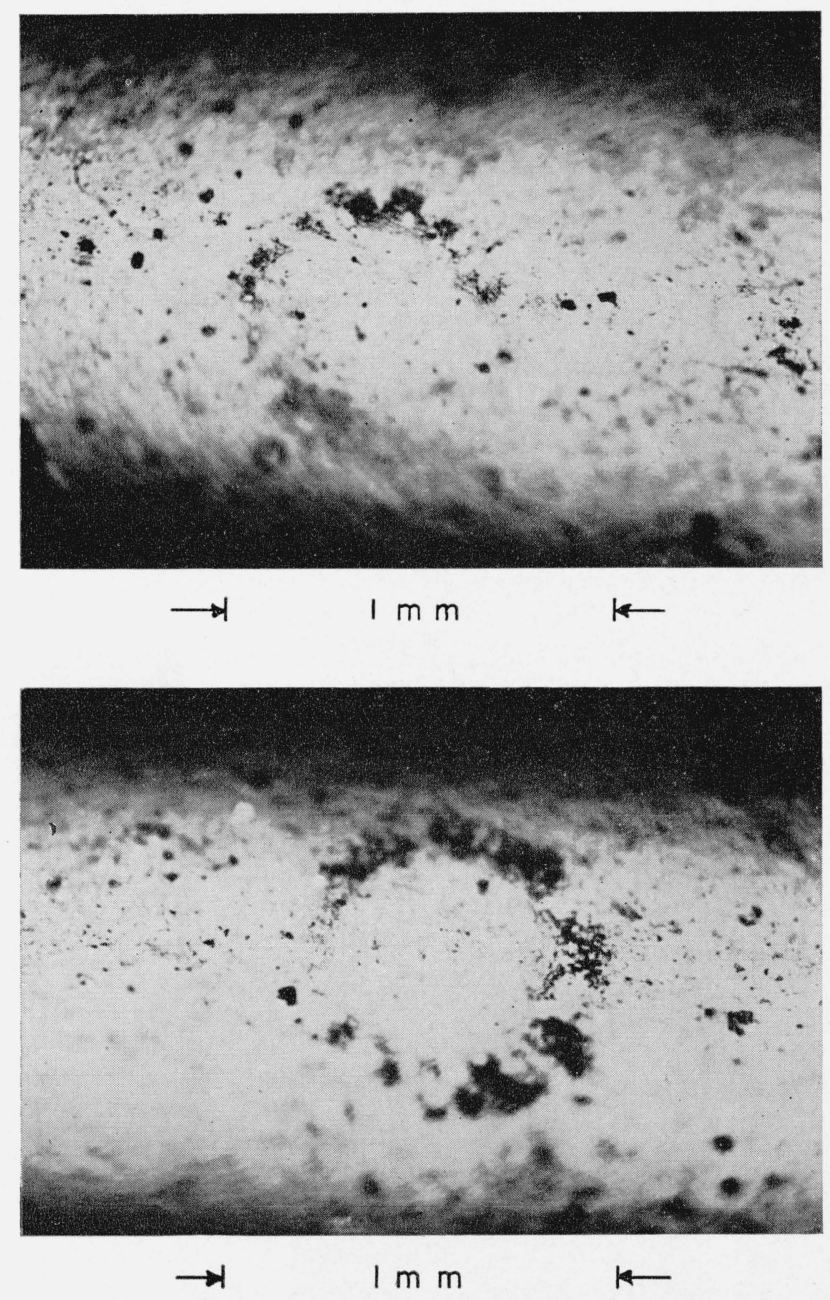

Figure 5. Circular damage marks produced on an experimental Neoprene coating that collided with waterdrops at a velocity of $880 \mathrm{fps}$.

\subsection{Waterdrop Traces in Polymeric Materials}

Figure 6 shows damage marks that were produced on four different polymeric materials by collision of the specimen material with waterdrops at a velocity of $2,250 \mathrm{fps}$. The experiment was carried out at Convair. The Neoprene coating was one of the standard Neoprene coatings that fulfilled the requirements of specification MIL-C-7439. Arrows in the picture point to the circular waterdrop traces. The undamaged centers, which mark the stagnation point of the radial flow, are very clear in the case of the two traces at the left of the picture. Circular damage traces were also produced in Teflon, Selectron 5003 polyester resin, and methyl methacrylate plastic. These pictures show that the forces exerted by the radial flow of a waterdrop as a result of collision with the planar surface of a solid at a velocity of $2,250 \mathrm{fps}$ are effective damage tools.

\section{Response of Structural Materials to High- Speed Waterdrop Impingement}

The damaging properties of an impinging liquid drop that have been considered in the preceding sections are similar for drops of all liquids but vary in intensity depending on the density of the liquid, on the relative collision velocity, and on the extent to which the solid material yields under the blow. If the characteristic properties of all structural materials were the same, the appearance of damage marks produced by the impingement of drops of a given liquid against all of them at some arbitrary velocity would also be the same; there would be only one type of damage and only one mechanism by which the damage is produced. Because the characteristic properties of structural materials are different, there are as many different damage processes as there are broad groups of material properties. A specific question that was posed at the time that the research program on the mechanism of highspeed rain erosion was initiated was whether a soft rubbery material or a hard rigid material should be sought as a solution to the rain-erosion problem. These two extreme cases are considered in the following sections.

\subsection{Rubberlike Yield}

A material that behaves like rubber under the blow that results from collision with a liquid drop has the advantage of being a pressure reducer for the blow. When the impact pressure is reduced, the velocity of the radial flow of the liquid of the drop, which is driven by the impact pressure, is also reduced. Because the radial flow velocity is reduced, the shear stress that it exerts, which decreases as the radial flow velocity decreases, is likewise reduced. The material is, however, depressed at the point where the liquid drop struck and radial tensile stresses appear in the sides of the cup-shaped depression and around and especially over the rim of it as it forms. The surface layers of the material around the restricted area of contact between the drop and the surface of the solid are also stretched as a result of the rapid flow of liquid from the drop. A material of this kind need only have strength properties sufficient to withstand the mitigated stresses in order to remain undamaged on colliding with a liquid drop. 


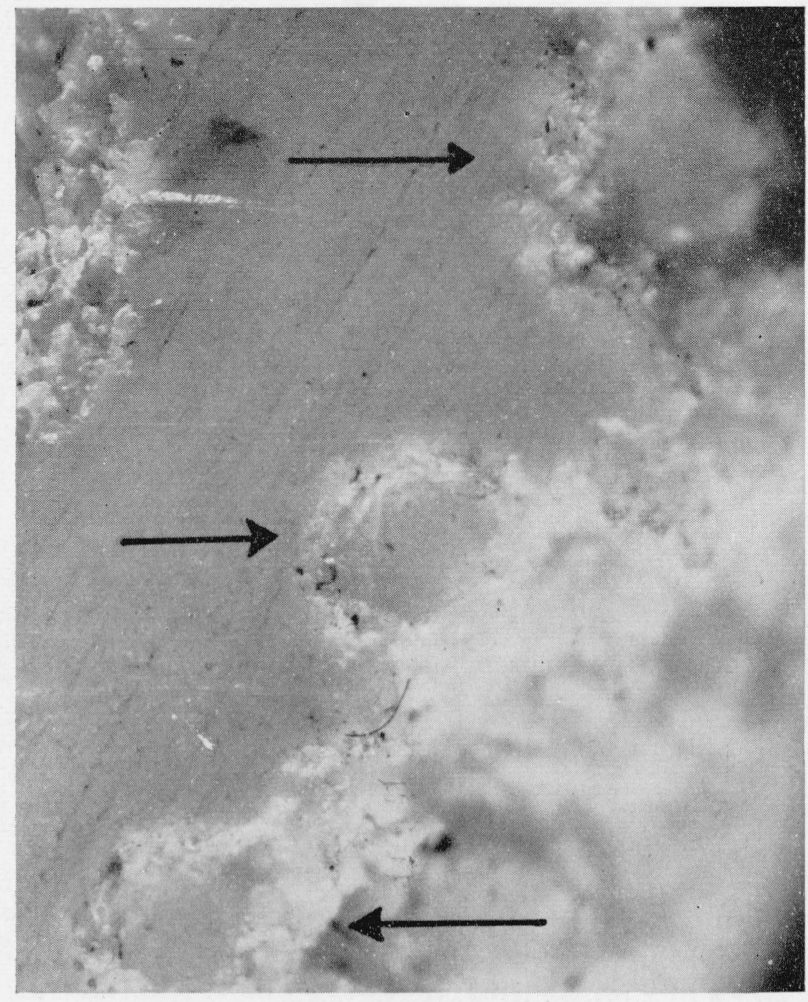

TEFLON

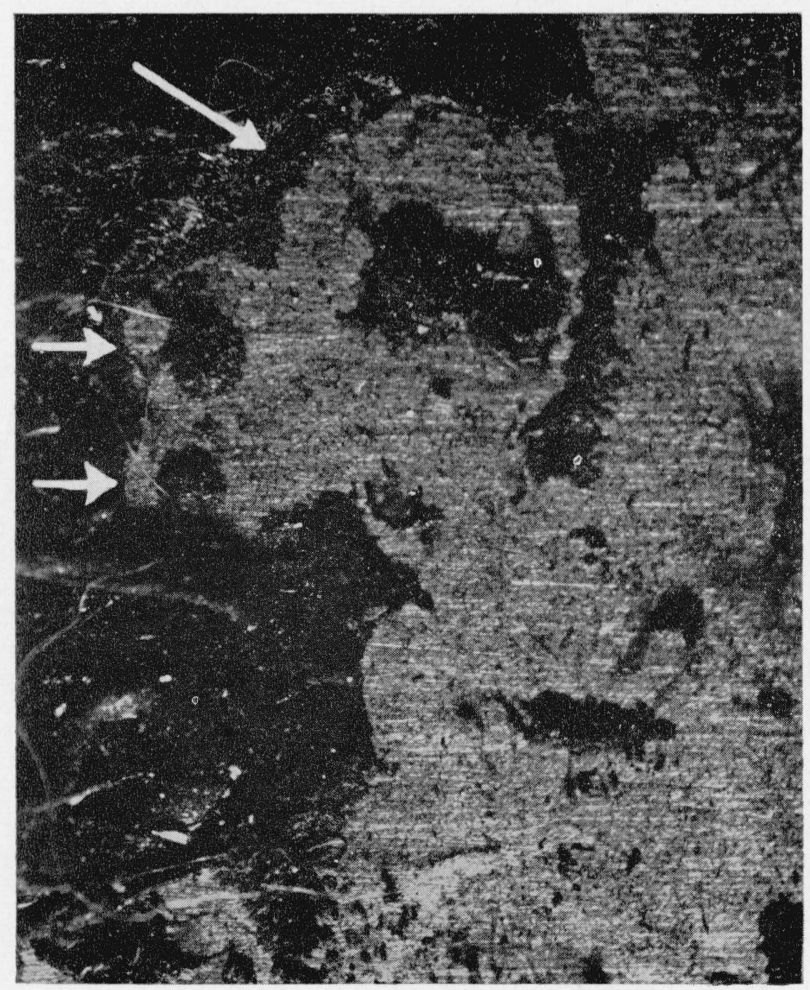

NEOPRENE

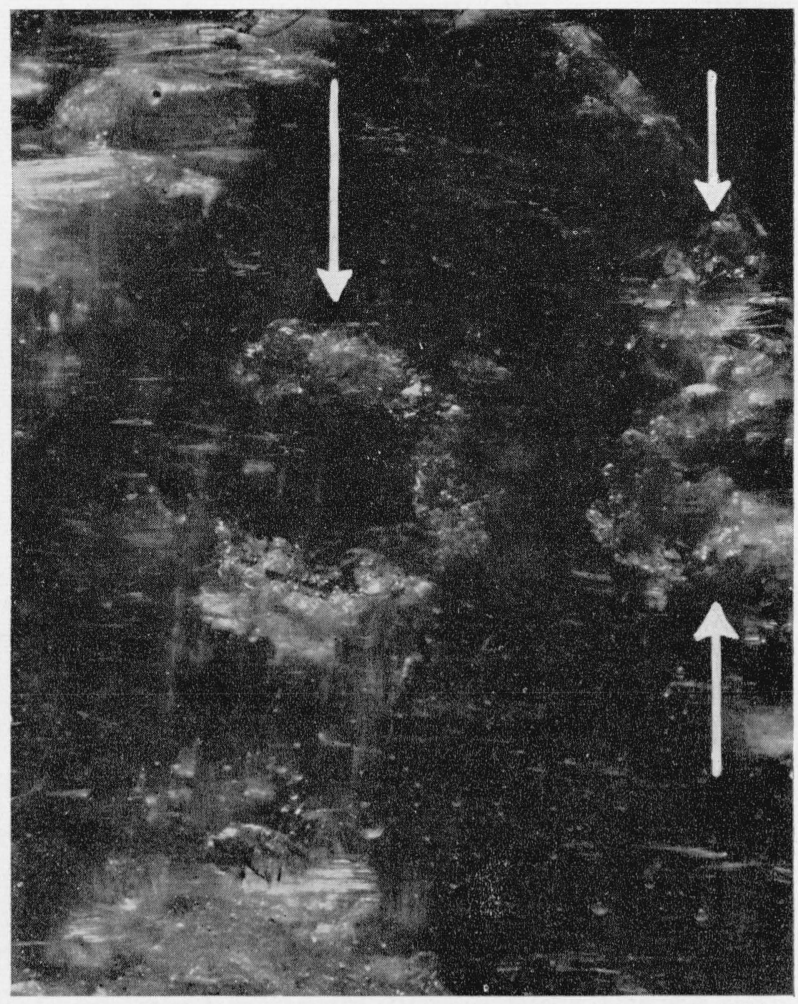

SELECTRON 5003

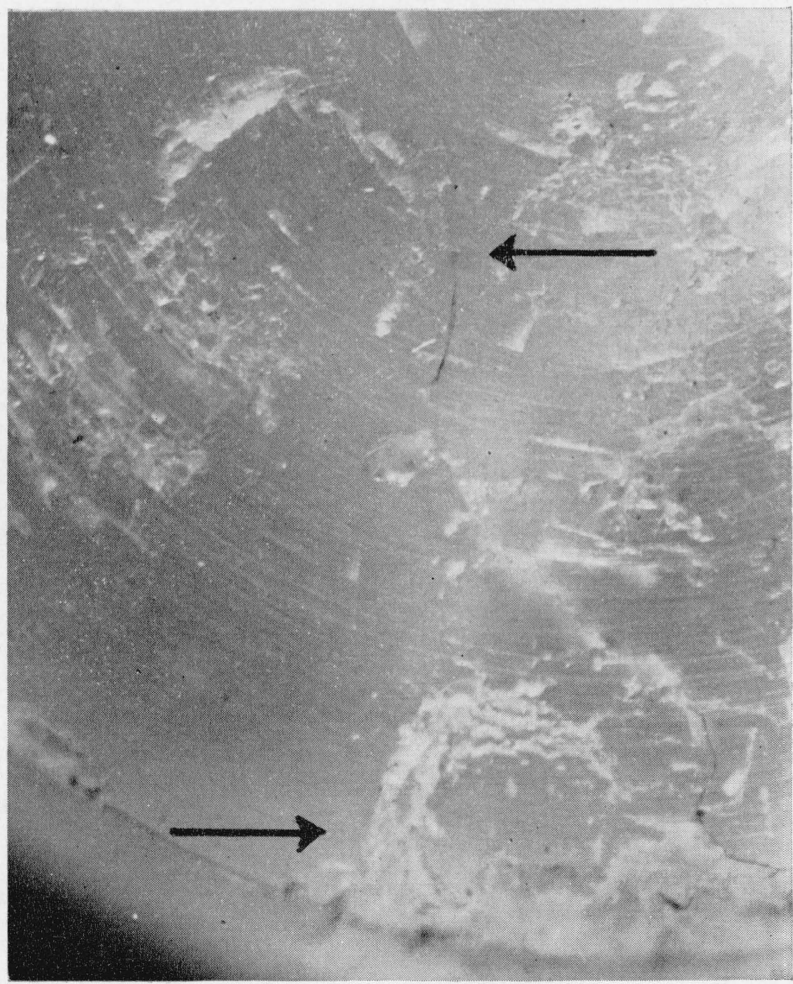

METHYL METHACRYLATE

Figure 6. Circular damage marks that were produced by collision with waterdrops at a velocity of 2,250 fps. 
Increase in the indentation hardness of a rubber is commonly accompanied by a decrease in its elongation under a given stress and, therefore, by a decrease in its ability to mitigate the stresses that are imposed on it by high-speed collisions with a waterdrop. The indentation hardness associated with maximum resistance against waterdrop-impingement damage at an arbitrary collision velocity is that hardness above which the gain in strength that often accompanies further hardening is unable to offset the loss in ability to mitigate the stresses that are imposed by collision with a waterdrop at the velocity in question. In terms of the rupture energy per unit volume, the optimum indentation hardness is that hardness above which the area under the stress-strain curve begins to decrease.

\subsection{Resistance of Hard Materials}

All materials yield to some extent under a collision blow. However, materials that do not display a high elongation under a given stress as a result of collision with a liquid drop do not mitigate to a notable degree the stresses that the colliding drop exerts. To be erosion resistant, materials of this class must be able to withstand the unmitigated stresses. Whether or not they can withstand the unmitigated stresses depends on whether they can absorb the energy of the collision before their yield or fracture strength is exceeded. If the relative collision velocity between the solid material and the liquid drop is increased without limit all the known hard materials will be found to fail at the point where their yield or fracture strength is exceeded; they will either yield with plastic flow or they will shatter.

Poly(methyl methacrylate) is a practical, rigidmaterial solution to the rain-erosion problem for flight velocities up to $300 \mathrm{mph}$. When specimens of poly (methyl methacrylate) are rotated through 1-iph simulated rain at a velocity of $300 \mathrm{mph}$, initial pitting of the plastic occurs after $45 \mathrm{~min}$. If, however, the velocity is increased to $400 \mathrm{mph}$, initial pitting occurs after $2 \mathrm{~min}$. Test results show that polystyrene and the glass-cloth-reinforced polyester resins are less resistant to rain erosion than poly(methyl methacrylate). The combined properties of rigidity and low strength eliminate these polymers as practical rain-erosion resistant materials at ordinary airplane flight velocities.

Metals undergo permanent plastic flow when their yield strength is exceeded. In the case of $1100-\mathrm{O}$ aluminum, the depression in the surface caused by a single collision with a waterdrop at a velocity of 600 $\mathrm{mph}$ is barely perceptible. The pummeling action of repeated collisions produces, by plastic flow, a general unevenness of the surface. This unevenness appears to play a role in enhancing a delayed, drastic failure of the material. Moderately thin specimen plates of the soft and medium hard metals undergo considerable plastic flow during a single collision with a waterdrop at velocities in the range of about 1,000 to $2,000 \mathrm{fps}$; the characteristic damage mark is a pit that resembles an empty spherical segment. See figure $1, \mathrm{C}$.
The hard metals are known to afford a practical solution to the high speed rain-erosion problem at moderately low impingement velocities. Incipient failure shows up only after hours of waterdrop impingement in the erosion test at a velocity of $500 \mathrm{mph}$ in a 1-iph rain density. The failure of the hard metals in this range of impingement velocity cannot be explained in terms of the impact pressure produced by collision with a waterdrop. It appears to involve pressure-raiser defects, such as microscopic pits and pores on the surface of the specimen, or weak spots in the material at or close to the surface, or both.

Of the materials that have been tested for resistance against collision with waterdrops at high relative impingement velocities, the ceramics have been found to be among the most resistant. However, if the impingement velocity is increased without limit, these materials also shatter at the point where their fracture strength is exceeded. A practical tensile strength of 30,000 psi can be expected from polycrystalline alumina compared to lower values for the soft metals and plastics.

\section{Requirements for Rain-Erosion Resistance}

The question that was posed several years ago as to whether the solution to the high-speed rainerosion problem should be sought among the soft rubbery materials or among the hard rigid materials can be answered in the following way. The material may be hard and rigid, or it may be soft and rubbery and still have a high degree of rain-erosion resistance. If it is hard and rigid, it must have strength properties that will enable it to withstand, without fracture and without plastic flow, the maximum unmitigated stresses imposed by collision with a waterdrop in the velocity range for which the material is being tested; these stresses increase in magnitude as the impingement velocity is increased. If it is soft and rubbery, it need only have strength properties sufficient to withstand the mitigated stresses that are imposed by collision with the waterdrop. The velocity ceiling for the rubbery materials is the point at which they are no longer able to withstand the mitigated stresses.

These remarks apply to the ability of a material to withstand a single waterdrop blow. Under actual flight conditions successive blows on the same spot have a certain probability of being very closely spaced in time. Therefore, under flight conditions a rain-erosion resistant rubbery material must not only have strength properties adequate to withstand the mitigated stresses but must also recover fast enough to be able to mitigate the stresses of an additional blow to the extent that they do not exceed its strength properties. To be a practical rain-erosion resistant material the rubber must, furthermore, not lose its ability to mitigate the stresses through deterioration of any kind during its expected service life.

Washington, April 9, 1957. 